\title{
Inverse Cascades Sustained by the Transfer Rate of Angular Momentum in a 3D Turbulent Flow
}

\author{
Miguel López-Caballero* and Javier Burguete ${ }^{\dagger}$ \\ Departamento de Física y Matemática Aplicada, University of Navarra, P.O. Box 177, E-31080 Pamplona, Spain
}

(Received 16 May 2012; published 20 March 2013)

\begin{abstract}
The existence of energy cascades as signatures of conserved magnitudes is one of the universal characteristics of turbulent flows. In homogeneous 3D turbulence, the energy conservation produces a direct cascade from large to small scales, although in $2 \mathrm{D}$, it produces an inverse cascade pointing towards small wave numbers. In this Letter, we present the first evidence of an inverse cascade in a fully developed 3D experimental turbulent flow where the conserved magnitude is the angular momentum. Two counterrotating flows collide in a central region where very large fluctuations are produced, generating a turbulent drag that transfers the external torque between different fluid layers.
\end{abstract}

DOI: 10.1103/PhysRevLett.110.124501

PACS numbers: 47.27.De, 47.27.wj

In his seminal work of 1941 [1,2], Kolmogorov postulated a mechanism for the transfer of energy from the injection scales towards the small scales, where it is finally dissipated [3]. This direct cascade with the now classical exponent of $-5 / 3$ has been verified in very different $3 D$ flows, whether homogeneous or not. But there are many topics that remain open; one of these is the creation and origin of coherent structures, with typical scales usually much larger than the injection scales. In 2D turbulence [4] where the vortices cannot be stretched, Kraichnan [5] proposed a new cascade in 1967: he introduced the concept of an inverse cascade where the energy was transported towards the large scales, whereas a direct cascade transported enstrophy to the small scales. Other particular cases of inverse cascades have been found in some special configurations, as in quantum fluids [6], in wave turbulence $[7,8]$, through the coupling of different modes in a Korteweg-de Vries model [9], in the transport of helicity in inviscid fluids [10,11], or inspired on the magnetic field generation in turbulent flows, the anisotropic kinetic alpha effect [12].

We analyze the behavior of a fluid in a closed cavity where two inhomogeneous and strongly turbulent flows collide in a thin region. Depending on the spatial position, different cascades have been found. Far from the collision layer, a classical Kolmogorov scenario is found, but in the shear region, inverse cascades appear. These cascades, that have been observed both in spatial and temporal spectra, correspond to the conservation of the axial angular momentum. A similar behavior may be present in any situation where large coherent structures are relevant and appear on the top of very turbulent colliding flows, as, for example, in atmospheric circulations [13], large scale currents and vortices in oceans [14], MRI instabilities and accretion disks [15-17], dynamo action in MHD [18,19], mixing problems [20], and industrial applications, to name a few.
Experimental setup.-The experimental volume is a closed cylinder with diameter $D=20 \mathrm{~cm}$ [see Fig. 1(a)]. Two impellers of diameter $D_{\text {prop }}=17.5 \mathrm{~cm}$ with 10 curved blades $[2 \mathrm{~cm}$ tall and $4.85 \mathrm{~cm}$ of curvature radius, see Fig. 1(c)] are located at $L=6 \mathrm{~cm}$ from both cylinder ends being the distance between them $H=20 \mathrm{~cm}$. The (a)

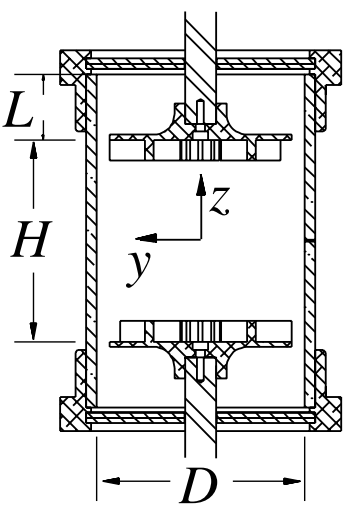

(c)

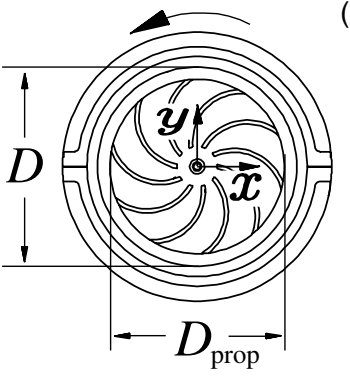

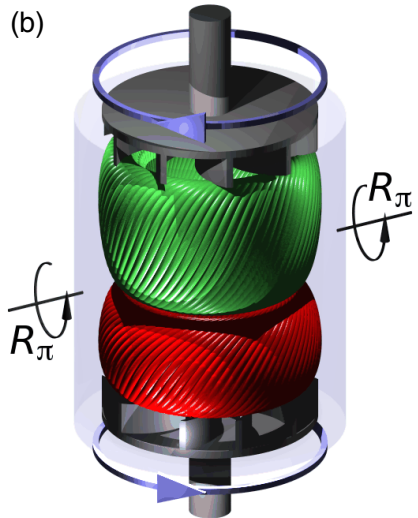

(d)

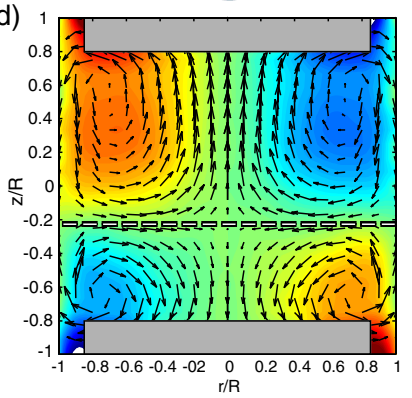

FIG. 1 (color online). Experimental setup: (a) Side view and (c) section of the cylindrical container. (b) Reconstruction of the asymmetric mean flow using averaged trajectories. (d) Mean flow in a meridional plane (the gray zones correspond to the propellers): Contour plot of $v_{\theta}$ (red means outwards, blue inwards) and vector field of $v_{z}$ and $v_{r}$. The white dashed line indicates the position of the shear layer $z_{\mathrm{sh}}=-22 \mathrm{~mm}$. The arrows in $(b, c)$ indicate the impellers rotation directions. 
disks rotate in opposite directions and are powered by two independent motors (maximum power of $1.5 \mathrm{~kW}$ ) regulated through a computer. The rotation frequency of the propellers can be adjusted independently between $f=$ $0.5-12.5 \mathrm{~Hz}$ generating a maximum torque of $15 \mathrm{Nm}$. A key characteristic of this setup is the high inertia of the propeller and motor set and the high stability of the propellers; i.e., the instantaneous fluctuations of each one of the propeller's velocities are well below one part in one thousand, $0.1 \%$. The cylinder is placed inside a square tank of $150 \mathrm{l}$ of volume filled with water at $21^{\circ} \mathrm{C}$ to reduce optical distortions and to ensure a high thermal inertia.

We have used two different techniques to measure the components of the velocity field (radial $v_{r}$, azimuthal $v_{\theta}$, and axial $v_{z}$ ). The first one is a 1D Laser Doppler Anemometry (LDA) system, with a measurement volume of $1.3 \times 0.3 \times 0.3 \mathrm{~mm}^{3}$, a spatial resolution of $1 \mathrm{~cm}$ and a temporal resolution up to $100 \mathrm{kHz}$. The second technique is a PIV system using a Nd-YAG pulsed laser and a $1600 \times$ 12001 inch CCD camera with an acquisition frequency up to $15 \mathrm{~Hz}$. Three different fields of view have been used to achieve this goal, $F_{1}=155 \times 125 \mathrm{~mm}, F_{2}=$ $55 \times 41 \mathrm{~mm}$, and $F_{3}=17.5 \times 13 \mathrm{~mm}$. We have used $32 \times 32$ px interrogation windows with a $50 \%$ overlapping and no offset. The tracer particles used in the presented data are silver coated hollow glass spheres (diameter $14 \mu \mathrm{m}$ and density $\rho=1.65 \mathrm{~g} / \mathrm{cm}^{3}$ ) because of their high reflectivity. The corresponding Stokes number for these particles is $\mathrm{St}=0.02 \ll 1$ [21]. The Reynolds number is defined as $\operatorname{Re}=R V_{\text {prop }} / \mu$ using the rim velocity of the propellers $V_{\text {prop }}=\pi D_{\text {prop }} f$.

Results.-The averaged flow that appears in this configuration for large Re can be summarized by the sketch presented in Fig. 1(b). The flow is in a fully developed turbulent regime (Reynolds number up to $\mathrm{Re}=7 \times 10^{5}$ ), with a typical turbulent intensity of $50 \%$, and breaks the symmetry $\left(R_{\pi}\right)$ of the basic configuration, a $\pi$-rotation around any equatorial diameter. This symmetry breaking appears through a bifurcation from a symmetric flow for $\operatorname{Re}=10^{4}[22]$. The trajectories presented in red (respectively green) correspond to the average displacement of fluid elements in the flow produced by the bottom (respectively top) impeller. Both large toroidal trajectories have the same helicity. The separation layer between both flows is displaced from the equator [Fig. 1(d)], either to $z=+z_{\mathrm{sh}}$ or $z=-z_{\mathrm{sh}}$ and thus, two symmetric solutions are accessible for the same Re, each one being the result of the $R_{\pi}$ operation over the other [see Fig 1(b)]. The system jumps randomly and spontaneously between these two solutions, and this can be recovered from the evolution of the azimuthal velocity at the equator [Fig. 2(a)]. The power spectra of the different velocity components have been computed [Fig. 2(b)], and various power laws for different frequencies ranges and in different spatial locations have been found. Here, we will focus on the behavior of the slow (a)

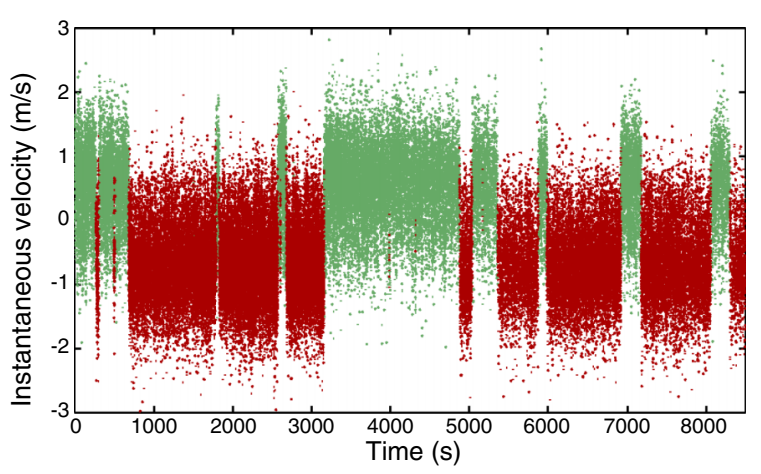

(b)

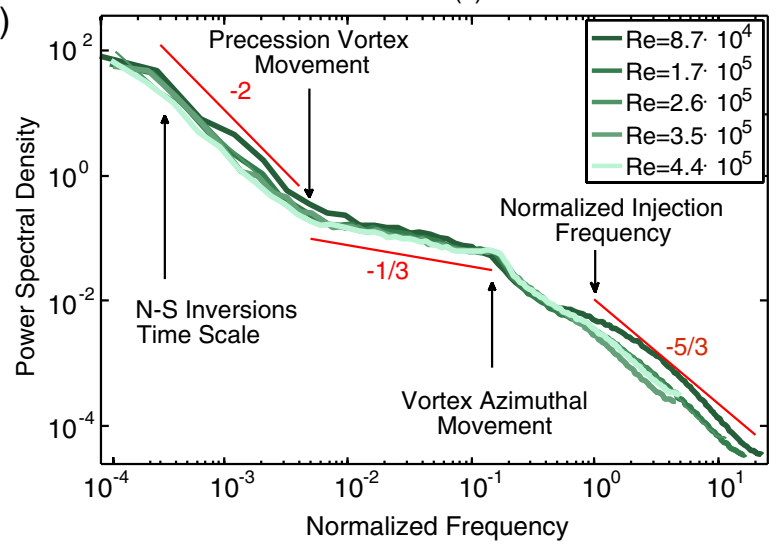

FIG. 2 (color online). Long term dynamics. (a) Azimuthal velocity near the wall $(r=0.9 R, z=0)$. The red (dark grey), respectively green (light grey) color indicates whether the south (respectively north) cell dominates [same color code as used in Fig. 1(b)]. (b) Spectra for different Re at this position.

scales below the injection frequency, where the fluctuations are very important and that appear mainly near the shear layer. This layer effectively constitutes a separation frontier between both averaged tori [red and green in Fig. 1(b)] as in average there is no transfer of mass across this surface. Although some of the characteristics have been observed in similar experiments [22-24], the whole picture has not been presented before.

The mean features of the turbulent flow have been determined. For a $\operatorname{Re}=1.75 \times 10^{5}$, the power dissipation rate is $\epsilon=1.1 \mathrm{~W} / \mathrm{kg}$. It has been obtained in two independent ways, using the second order structure function from PIV measurements and the third order structure function from high rate LDA series [25]. The dissipative scale is $\eta=31 \mu \mathrm{m}$, that corresponds roughly to twice the size of our tracers. From the cross correlation analysis of PIV velocity maps, we got an integral scale of $L_{I}=15 \mathrm{~mm}$. From the rms fluctuations, we derived a Taylor microscale of $\lambda_{T}=1.8 \mathrm{~mm}$, and the corresponding Reynolds number is $\operatorname{Re}_{\lambda}=900$.

Three different slopes can be identified in the log-log plot of the power spectra [see Fig. 2(b)]. These slopes are better defined for large values of the Reynolds number [26]. The classical value for a Kolmogorov cascade $(-5 / 3)$ appears for frequencies larger than the injection 
frequency. The slope -2 corresponds to the reversals or inversions of the averaged flow and appears for extremely low frequencies (between 2 and 4 orders of magnitude below the injection times). That corresponds to a dynamical behavior, not really an energy cascade, and has been analyzed elsewhere $[22,23]$. These reversals can be easily controlled and even removed as explained in Ref. [23]. We have verified that the high stability of the propeller's velocities plays a key role on this dynamics: artificially introduced fluctuations larger than $1 \%$ (through function generators) $[22,23]$ are enough to destroy the presented slow scales, so this can explain why this behavior has not been observed in other setups, where typical values of the propeller fluctuations are 1-3\%. In the following, all the presented data will correspond to the behavior of one of the discussed states, as the one plotted in Fig. 1(b) and 1(d), without reversals.

In the intermediate region between the injection frequency and the reversals, another cascade with a slope $-1 / 3$ is identified, that extends over 2 orders of magnitude. This cascade only appears in a region at both sides of the shear layer covering roughly $30 \%$ of the total height $\mathrm{H}$, and disappears when we approach the impellers. In the shear layer, we can observe the creation of coherent structures that lie near the wall [see the inset in Fig. 3(a)]. Those

(a)

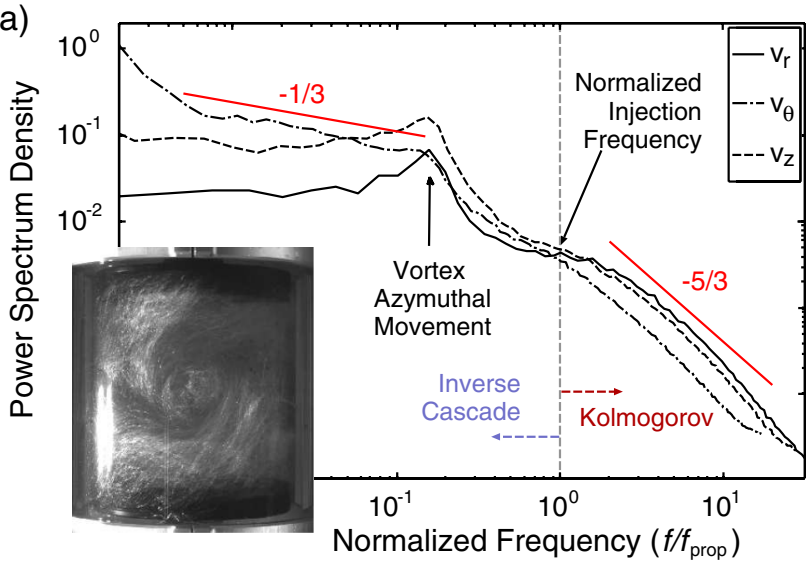

(b)

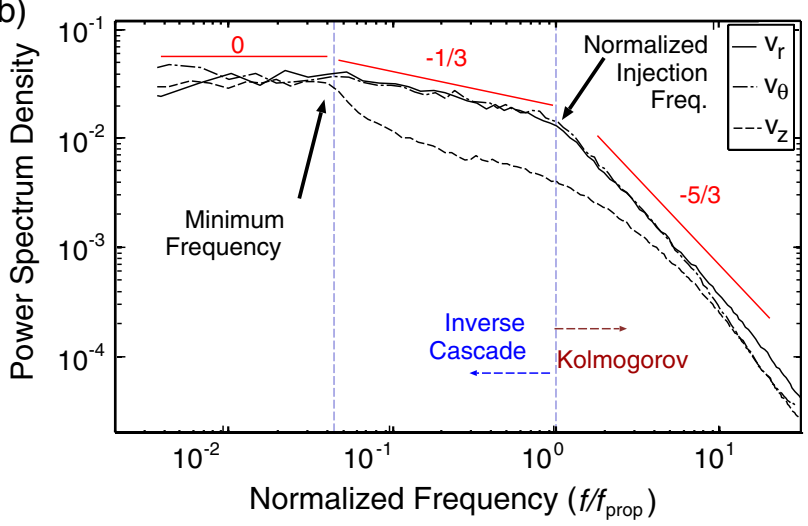

FIG. 3 (color online). Power spectra computed from LDA data series (a) near the wall $r=0.9 R, z=0$ and (b) at the center $r=0$, $z=0$. Both spectra present direct and inverse cascades. vortices are responsible for the different behavior of the corresponding temporal spectra for different radial positions. Near the wall, a maximum appears because of these coherent structures [Fig. 3(a) and inset], and the slope is displaced towards lower frequency values. Close to the axis, the behavior is different, as there is a clear $-1 / 3$ slope without peaks [see Fig. 3(b)]. This cascade doesn't continue, however, indefinitely towards the slow scales, but there is a minimum frequency [Fig. 3(b)] that corresponds to the maximum time scale observed in the dynamics (without reversals).

Any cascade that appears in a flow can be the signature of a conserved magnitude. The conservation of angular momentum in turbulent flows was proposed many years ago [27] and different groups have verified [28,29] that it is a constant of motion. For large Re, the viscous terms cannot dissipate this magnitude, so the torque injected by one propeller produces a drag between different layers of the flow being finally transferred to the other propeller. Using the same approach presented in Ref. [28], the balance of the axial component of the angular momentum $\mathcal{L}$ can be computed, for a thin slice of the experimental volume perpendicular to the axis, as the difference of the drag between the upper and bottom layers (we neglect the contribution or losses on the lateral wall),

$$
\begin{aligned}
\partial_{t}\left\langle\mathcal{L}_{V}\left(z_{0}\right)\right\rangle= & {\left[\int_{V} \partial_{t}\langle\mathcal{L}\rangle d V\right]=\left[\int_{V} \rho\left\langle r v_{z} \partial_{z} v_{\theta}\right\rangle d V\right]_{z_{0}} } \\
= & {\left[\int_{A} \rho\left\langle r u_{\theta} u_{z}\right\rangle d A+\int_{A} \rho r U_{\theta} U_{z} d A\right]_{z_{0}+\Delta z / 2} } \\
& -\left[\int_{A} \rho\left\langle r u_{\theta} u_{z}\right\rangle d A+\int_{A} \rho r U_{\theta} U_{z} d A\right]_{z_{0}-\Delta z / 2},
\end{aligned}
$$

where $\mathcal{L}_{V}\left(z_{0}\right)$ measures the $z$ component of the angular momentum per unit mass in the considered volume $V=$ $A \Delta z$ between $z_{0}-\Delta z / 2$ and $z_{0}+\Delta z / 2$ and where $A=$ $\pi R^{2}$. The velocities have been decomposed in two terms $\vec{v}=\vec{u}+\vec{U}$, being $\vec{U}$ the mean value and $\vec{u}$ the zero-mean fluctuations where the slow and fast scales appear combined.

We can define the magnitude $\mathcal{T}(r, z)=r v_{\theta} v_{z}$ as the torque transferred per unit area along the axis. We have computed the temporal evolution of this magnitude and the corresponding cospectrum (see Fig. 4) for different positions $(r, z)$. We can observe similar cascades with the same slopes to those observed in the power spectra. From this data, we can obtain the total torque $\mathcal{T}_{A}=\int_{A} \mathcal{T} d A$ transferred through the surface $A$ for different $z$. This magnitude is constant along $z$, so the torque balance across a fluid slice is zero (i.e., the drag going into a layer balances the one going out) and the angular momentum is conserved. At each surface, the drag can be transferred either by the slow fluctuations $\mathcal{T}_{A}^{u}$ or by the mean flow $\mathcal{T}_{A}^{U}$, so $\mathcal{T}_{A}=\mathcal{T}_{A}^{u}+$ $\mathcal{T}_{A}^{U}$. Depending on $z$, one term or the other is dominant: 


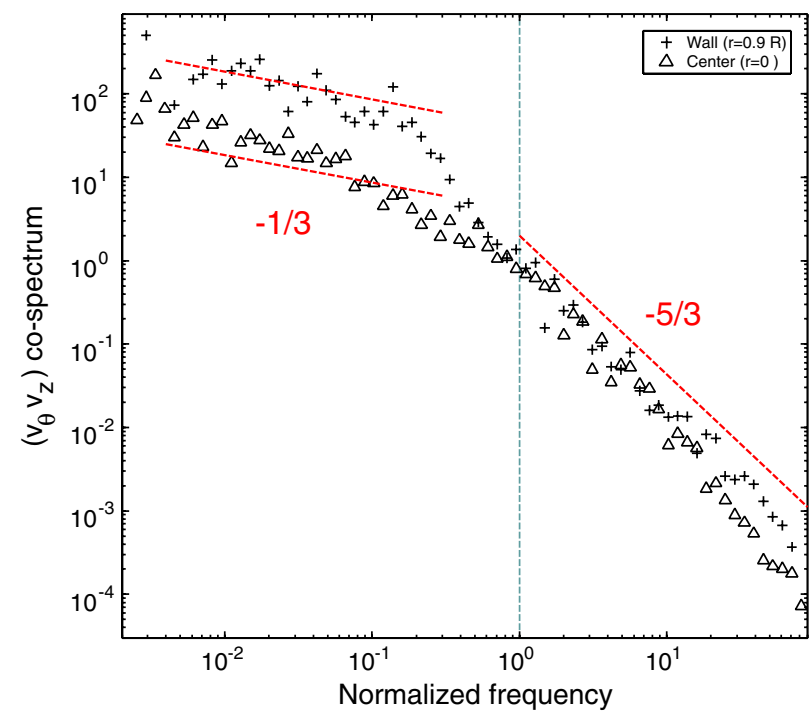

FIG. 4 (color online). Cospectrum of $v_{z} v_{\theta}$ computed using LDA data from series $10^{3}$ seconds long at two different spatial positions $r=0$ and $r=0.9 R$. Two regimes are clearly differentiated, a direct cascade starting from the injection frequency and an inverse cascade towards the slow scales.

near the shear layer, where $U_{z} \simeq U_{\theta} \simeq 0$, only the fluctuations transfer this torque $\mathcal{T}_{A}=\mathcal{T}_{A}^{u}$. When we compute $\mathcal{T}_{A}$ close to the propellers, where the mean velocity $U$ is important, the torque is transferred mainly by the mean flow $\mathcal{T}_{A}^{U} \simeq 5 \mathcal{T}_{A}^{u}$. Moreover, we can split $\mathcal{T}_{A}^{u}$ into two parts, corresponding to time scales faster $\mathcal{T}_{A}^{u^{>}}$and slower $\mathcal{T}_{A}^{u^{<}}$than the injection scales. The computation of each one of these contributions from the data shown in Fig. 4, reveals that the fast scales have a marginal contribution to the total transferred torque and can be neglected $\mathcal{T}_{A}^{u^{>}} \simeq 0$. Any effect of the torque fluctuations on the angular momentum should appear for frequencies smaller than the injection scale, just where the slopes are observed.

In order to verify the effect of these fluctuations, we can analyze the evolution of its axial component $\mathcal{L}$ at a given position as

$$
\partial_{t} \mathcal{L}_{V}^{2} \sim \int_{V} 2 r^{2} v_{\theta} v_{z} \partial_{z} v_{\theta} d V
$$

This magnitude defines the transfer of angular momentum. In a given position, the transfer rate of the angular momentum will evolve as $\varepsilon_{L} \propto L^{2}\left(L^{3} / T^{3}\right) / L=L^{4} / T^{3}$, where $L$ and $T$ designate, respectively, spatial and temporal scales. Coming back to the angular momentum contained in a given volume, for turbulent flows with isotropic and homogeneous fluctuations we obtain:

$$
\mathcal{L}_{V}^{2}=\int \mathcal{L}_{F}^{2}(k) d k
$$

where $\mathcal{L}_{F}^{2}(k)$ is the Fourier transform of $\mathcal{L}^{2}(r)$. If the angular momentum is a conserved magnitude, and transferred between scales in a wide range of wave numbers, we would expect that $\mathcal{L}_{F}^{2}(k)$ only depends on the transfer rate of angular momentum and on the wave number, through a law:

$$
\mathcal{L}_{F}^{2}(k) \propto \varepsilon_{L}^{2 / 3} k^{-7 / 3} .
$$

An analysis of the corresponding kinetic energy $\mathcal{E}_{V}=$ $\mathcal{L}_{V}^{2} / I_{V}$, where $I_{V}$ is the moment of inertia of the corresponding fluid layer around the axis, shows that the same scaling should be obtained for $\mathcal{E}$ :

$$
\mathcal{E}(k) \propto \mathcal{R}^{-2} \varepsilon_{L}^{2 / 3} k^{-7 / 3},
$$

where $\mathcal{R}$ is a characteristic length of the moment of inertia $I$. These spectra have been obtained from PIV measurements (Fig. 5). Two different regimes with two clear slopes are identified. The slope for the inverse cascade is $-7 / 3$ (see Fig. 5, inset, where the data are compensated with $k^{7 / 3}$ ) and the direct cascade is close to $-5 / 3$. This last value is in the limit of our resolution, as we have no access to smaller scales where the cascade continues (the wave number of the dissipation length $\eta$ is $k_{\eta} \simeq 200 \mathrm{~mm}^{-1}$ ). The integral scale (that is close to the flow modulation induced by the blades in the azimuthal direction) and the Taylor microscale wave numbers are indicated.

Moreover, a similar analysis shows that the temporal behavior for a given position scales as

$$
\mathcal{E}(\omega)=\varepsilon_{L}^{2 / 3} U^{-2 / 3} \omega^{-1 / 3},
$$

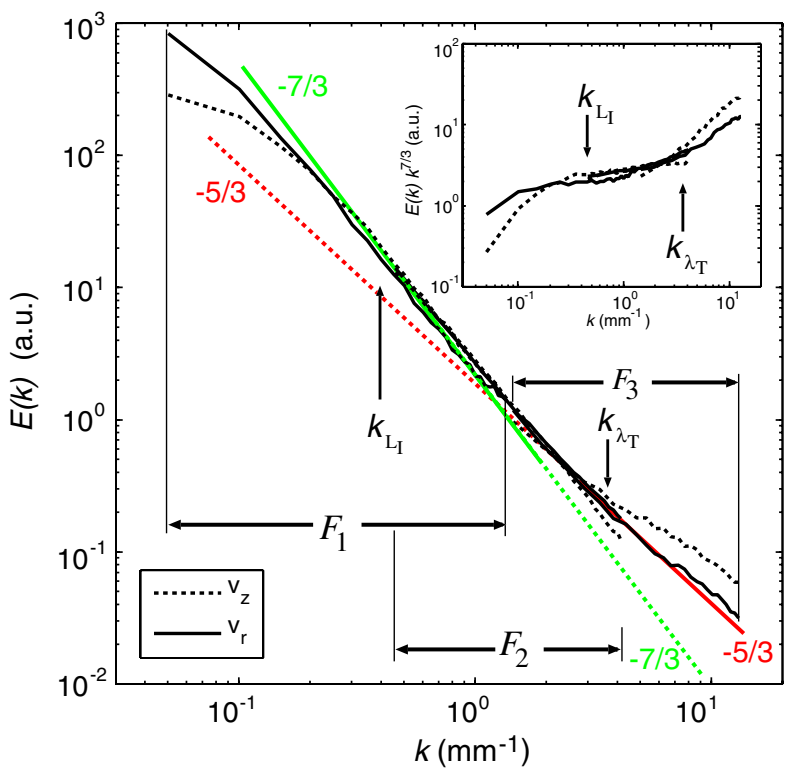

FIG. 5 (color online). Spatial spectra computed from PIV measurements. The wave number ranges covered by the different fields of view $F_{1}, F_{2}$, and $F_{3}$ are presented (the full range covers from 0.05 to $13 \mathrm{~mm}^{-1}$ ). Inset: Spectra compensated by $k^{7 / 3}$ where the slope change is clearly visible. $k_{L_{I}}$ and $k_{\lambda_{T}}$ correspond to the integral scale and the Taylor microscale. Each one of these spectra is the average of 1500 realizations along a diameter at $z=z_{\text {sh }}$ [dashed line in Fig. 1(d)]. 
where $U$ is the average flow velocity. So, we recover the behavior of the temporal spectra presented in Figs. 2-4.

Conclusions.-We have presented for the first time a fully developed 3D turbulent flow where an inverse cascade appears in the angular momentum $\mathcal{L}^{2}$ due to the transport of the external torque. This scenario where inverse cascades transport angular momentum between different scales is expected in other configurations. The candidate flows should have a turbulent shear layer where the fluctuations are dominant and an interface between two well-defined averaged flows where there is a transport of torque.

We thank A. de la Torre, W. Gonzalez-Viñas, J.F. Pinton, M. Bourgoin, R. Volk, and A. Pumir for fruitful discussions. This research was supported by Spanish Government through Contracts No. FIS2008-01126 and No. FIS2011-24642. M. L.C. thanks the "Asociacion de Amigos" from University of Navarra for financial support.

*mlcdos@gmail.com

†javier@unav.es

[1] A. N. Kolmogorov, Proc. R. Soc. A 434, 9 (1991).

[2] A. N. Kolmogorov, Proc. R. Soc. A 434, 15 (1991).

[3] S. B. Pope, Turbulent Flows (C. U. Press, Cambridge, 2000).

[4] P. Tabeling, Phys. Rep. 362, 1 (2002).

[5] R. H. Kraichnan, Phys. Fluids 10, 1417 (1967).

[6] J. Salort et al., Phys. Fluids 22, 125102 (2010).

[7] V. E. Zakharov, V. S. L'vov, and G. Falkovich, Kolmogorov Spectra of Turbulence I-Wave Turbulence (SpringerVerlag, Berlin, 1992).

[8] V.E. Zakharov, F. Diasc, and A. Pushkarev, Phys. Rep. 398, 1 (2004).
[9] A. Hasegawa, Y. Kodama, and K. Watanabe, Phys. Rev. Lett. 47, 1525 (1981).

[10] E. Levich and E. Tzvetkov, Phys. Rep. 128, 1 (1985).

[11] E. Levich and A. Tsinober, Phys. Lett. 93A, 293 (1983).

[12] U. Frisch, Z.S. She, and P.L. Sulem, Physica (Amsterdam) 28D, 382 (1987).

[13] M. V. Kurgansky, Phys. Chem. Earth 24, 959 (1999).

[14] A. Provenzale, A. Babiano, A. Bracco, C. Pasquero, and J. Weiss, Lect. Notes Phys. 744, 101 (2008).

[15] H. Ji, M. Burin, E. Schartman, and J. Goodman, Nature (London) 444, 343 (2006).

[16] M.S. Paoletti and D. P. Lathrop, Phys. Rev. Lett. 106, 024501 (2011).

[17] D. P. M. van Gils, S. Huisman, G. W. Bruggert, C. Sun, and D. Lohse, Phys. Rev. Lett. 106, 024502 (2011).

[18] A. de la Torre, J. Burguete, and C. Perez-Garcia, Eur. Phys. J. Special Topics 146, 313 (2007).

[19] A. Giesecke, F. Stefani, and J. Burguete, Phys. Rev. E 86, 066303 (2012).

[20] P.E. Dimotakis, Annu. Rev. Fluid Mech. 37, 329 (2005).

[21] H. Xu and E. Bodenschatz, Physica (Amsterdam) 237D, 2095 (2008).

[22] A. de la Torre and J. Burguete, Phys. Rev. Lett. 99, 054101 (2007).

[23] J. Burguete and A. de la Torre, Int. J. Bifurcation Chaos Appl. Sci. Eng. 19, 2695 (2009).

[24] F. Ravelet, A. Chiffaudel, and F. Daviaud, J. Fluid Mech. 601, 339 (2008).

[25] R. A. Antonia, T. Zhou, and G. P. Romano, Phys. Fluids 9 , 3465 (1997).

[26] Y. Zhou, Phys. Plasmas 14, 082701 (2007).

[27] L. Landau and E. Lifchitz, Fluid Mechanics (AddisonWesley, Reading, 1959).

[28] L. Marie and F. Daviaud, Phys. Fluids 16, 457 (2004).

[29] H. Xu, A. Pumir, and E. Bodenschatz, Nat. Phys. 7, 709 (2011). 